\title{
Aneurysmal Degeneration of the Brachial Artery after Vascular Access Creation: Surgical Treatment Results
}

\author{
Sérgio Teixeira, $\mathrm{MD}^{1}$ Pedro Sá Pinto, $\mathrm{MD}^{1}$ Carlos Veiga, $\mathrm{MD}^{1} \quad$ Ivone Silva, MD, $\mathrm{PhD}^{1}$ \\ Rui Almeida, $\mathrm{MD}^{1}$ \\ ${ }^{1}$ Department of Angiology and Vascular Surgery, Centro Hospitalar \\ do Porto - Hospital de Santo António, Porto, Portugal \\ Address for correspondence Sérgio Oliveira Teixeira, MD, Serviço de \\ Angiologia e Cirurgia Vascular, Centro Hospitalar do Porto - Hospital \\ de Santo António, Largo Prof. Abel Salazar, 4099-001 Porto, Portugal \\ Int J Angiol 2017;26:186-190. \\ (e-mail: u10738@chporto.min-saude.pt).
}

Abstract

Keywords
- arteriovenous fistula
- brachial artery
aneurysm
- hemodialysis
- aneurysm
- brachial artery
- fistula
- vascular access

True peripheral artery aneurysms proximal to a longstanding arteriovenous fistula is a well-recognized complication. Late aneurysmal degeneration is rare. This study analyzed the characteristics, therapeutic options, and outcomes of true donor brachial artery aneurysms (DBAA) after arteriovenous fistula (AVF) for hemodialysis. We retrospectively collected the data of patients with DBAA after AVF creation, surgically repaired between January 2001 and September 2015. We excluded patients with pseudoaneurysms, anastomotic aneurysms, and infected aneurysms. We recorded patient's demographics, type of access, aneurysm characteristics, symptoms, treatment, and follow-up. Ten patients were treated for aneurysmal degeneration of the brachial artery. Average aneurysm diameter was $37.5 \mathrm{~mm}$. All cases had, at least, one previous distal AVF, ligated or thrombosed, at the time of diagnosis. The first access was created in mean 137 months before the diagnosis of DBAA. Nine patients had previous medical history of renal transplant and were under immunosuppressive therapy. All patients were symptomatic at the time of diagnosis. In all cases, the treatment was aneurysmectomy followed by interposition bypass. One patient developed a postoperative hematoma with the need of surgical drainage. At 50 months of follow-up, one patient was submitted to percutaneous angioplasty due to an anastomotic stenosis. No other complications occurred during the entire follow-up period (mean: 69 months). The pathogenesis underlying DBAA remains unclear. Increased blood flow after AVF creation, immunosuppressive therapy, and ligation/thrombosis of the AVF may contribute to aneurysm formation. Surgical treatment by aneurysmectomy and bypass, with autogenous conducts, is a safe and effective option.
Autologous arteriovenous fistulas (AVFs) are the preferred and recommended method for achieving vascular access for hemodialysis in patients with end-stage renal disease. ${ }^{1,2}$ First described by Hunter in 1757, arterial dilatation after longstanding AVF is a common finding as the artery adapts to the increased flow rate. ${ }^{3,4}$ Late aneurysmal degeneration of the donor arteries with the development of true aneurysms is rare. ${ }^{4}$ True aneurysms most frequently appear in the brachial artery but can also develop or involve other inflow arteries. $^{4-6}$ Aneurysmal degeneration is not prevented by closure or thrombosis of the arteriovenous access and may be associated with a history of renal transplantation and immunosuppressive treatment. ${ }^{4,5}$

Given their rarity, complexity, and late occurrence, there is no consensus on the management of donor brachial artery aneurysms (DBAA). ${ }^{6}$ In this study, we analyzed the characteristics, therapeutic options, and outcomes of true DBAA after AVF for hemodialysis. published online April 11, 2017
Copyright $\odot 2017$ by Thieme Medical Publishers, Inc., 333 Seventh Avenue, New York, NY 10001, USA. Tel: +1(212) 584-4662.
DOI https://doi.org/ 10.1055/s-0037-1601872. ISSN 1061-1711. 


\section{Methods}

Aneurysm is defined as an arterial enlargement $>50 \%$ of the normal diameter. The normal diameter for the brachial artery is 3.5 to $4.3 \mathrm{~mm}$ in women and 4.1 to $4.8 \mathrm{~mm}$ in men. ${ }^{7,8}$

We retrospectively reviewed the data of patients with DBAA after AVF creation, surgically repaired at the Centro Hospitalar do Porto - Hospital de Santo António, Porto, Portugal, between January 2001 and September 2015.

The study excluded patients with pseudoaneurysms, anastomotic aneurysms, and infected aneurysms. We recorded patient's demographics, type of access, aneurysm characteristics, symptoms, treatment, and follow-up.

Categorical data were described using absolute numbers and percentages. Means ( \pm standard deviation) and medians were used to analyze continuous variables.

\section{Results}

In this study, 10 patients ( 9 men [90\%] and 1 woman [10\%]) were treated for aneurysmal degeneration of the brachial artery after AVF creation, between January 2001 and September 2015 (-Table 1). Patients' mean age was $52 \pm$ 7.84 years (range: 37-63 years) at the time of treatment. Nine patients $(90 \%)$ had a previous history of renal transplantation and were under immunosuppressive therapy. Present cardiovascular risk factors were hypertension (90\% of patients), previous or current history of smoking (60\%), and dyslipidemia (50\%). No patients had diabetes.

All cases had at least one previous distal AVF. Five patients had 2 fistulas out of 15 fistulas in 10 patients. Patients with two AVFs, in four cases, after thrombosis/ligation of a radiocephalic fistula, created a brachiocephalic fistula and in one

Table 1 Summary of treated donor brachial artery aneurysms after AVF for hemodialysis

\begin{tabular}{|c|c|}
\hline Total number of cases & 10 \\
\hline Mean age \pm standard deviation & $52 \pm 7.84$ y (range: $37-63)$ \\
\hline Male & $9(90 \%)$ \\
\hline Female & $1(10 \%)$ \\
\hline \multicolumn{2}{|l|}{ Cardiovascular risk factors } \\
\hline Smoking (current/past) & $6(60 \%)$ \\
\hline Diabetes & 0 \\
\hline Hypertension & $9(90 \%)$ \\
\hline Dyslipidemia & $5(50 \%)$ \\
\hline Renal transplantation/immunosuppression & $9(90 \%)$ \\
\hline Time to the diagnosis (since AVF creation) & $136.7 \pm 60.4$ mo (range: $64-288$ ) \\
\hline Mean maximum diameter & $37.5 \pm 14.54 \mathrm{~mm}$ (range 17.5-64) \\
\hline \multicolumn{2}{|l|}{ Clinical presentation } \\
\hline Localized pain and pulsatile mass & $6(60 \%)$ \\
\hline Subacute ischemia/microembolization & $3(30 \%)$ \\
\hline Acute ischemia/aneurysm thrombosis & $1(10 \%)$ \\
\hline \multicolumn{2}{|l|}{ Previous ipsilateral AVFs } \\
\hline One & $5(50 \%)$ \\
\hline Two & $5(50 \%)$ \\
\hline \multicolumn{2}{|l|}{ Type of first access } \\
\hline Radiocephalic & $6(60 \%)$ \\
\hline Brachiocephalic & $4(40 \%)$ \\
\hline \multicolumn{2}{|l|}{ Adjuvant diagnostic modality } \\
\hline Computed tomography angiography & $3(30 \%)$ \\
\hline Conventional angiography & $2(20 \%)$ \\
\hline \multicolumn{2}{|l|}{ Treatment } \\
\hline Aneurysmectomy and GSV grafting & $6(60 \%)$ \\
\hline Aneurysmectomy and basilica vein grafting & $1(10 \%)$ \\
\hline Aneurysmectomy and cephalic vein grafting & $2(20 \%)$ \\
\hline Aneurysmectomy and ePTFE grafting & $1(10 \%)$ \\
\hline Mean follow-up & 69 mo (range: 1-177) \\
\hline
\end{tabular}

Abbreviations: AVF, arteriovenous fistula; ePTFE, expanded polytetrafluoroethylene; GSV, great saphenous vein. 


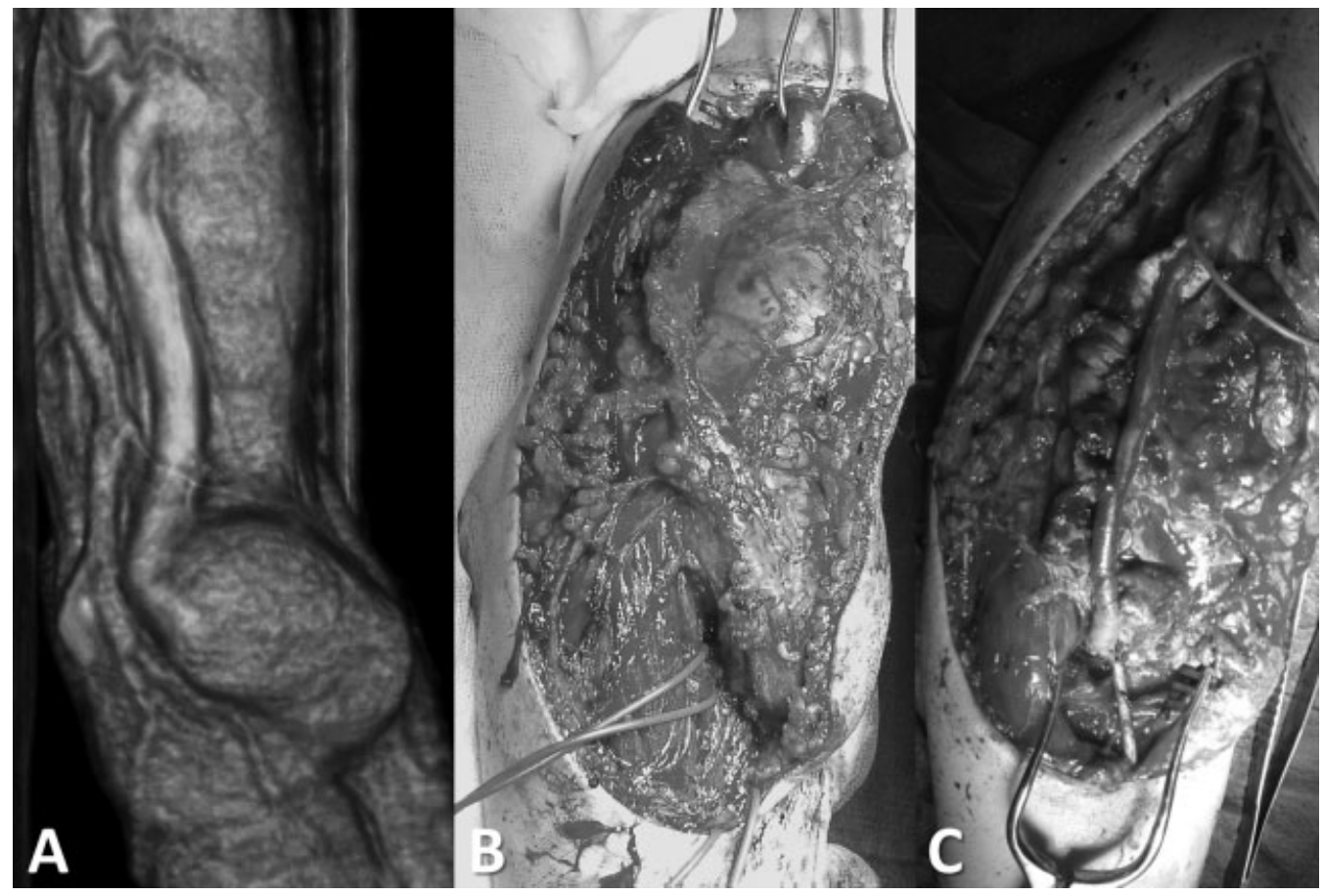

Fig. 1 (A) Preoperative CT scan (volume rendering image); (B and C) brachial artery dissection and brachiobrachial bypass with basilica vein. CT, computed tomography.

case, after thrombosis of a brachiocephalic fistula, created a brachiobasilic fistula. Five patients had one AVF (radiocephalic fistula-2 and brachiocephalic fistula-3). At the time of diagnosis, all the previous AVFs were occluded (thrombosis-4 and ligation-6).

The first access was created in mean $136.7 \pm 60.4$ months (range: 64-288 months) before the diagnosis of brachial artery aneurysm. Location of first access was radiocephalic in six patients and brachiocephalic in four.

All patients were symptomatic at the time of diagnosis: localized pain and pulsatile mass-6 (60\%), subacute ischemia/microembolization-3 (30\%), and acute ischemia/aneurysm thrombosis-1 (10\%). Duplex scan was the primary investigative modality in all cases. Computed tomography (CT) scan angiography and classic angiography were used only in selected cases. Mean aneurysm diameter was $37.5 \pm$ $14.54 \mathrm{~mm}$ (range: 17.5-64 mm). No case presented with multiple separate aneurysms (-Figs. 1-3).

In all cases, the treatment was aneurysmectomy followed by interposition bypass, using great saphenous vein-6, ipsilateral cephalic vein-2, ipsilateral basilica vein-1, or expanded polytetrafluoroethylene-1. One patient developed a postoperative hematoma with the need of surgical drainage. No other major complications occurred before discharge. At 50 months of follow-up, one patient underwent percutaneous angioplasty due to an anastomotic stenosis. No other complications occurred during the entire follow-up period (mean: $69.2 \pm 57.67$; range: $1-177$ months).

\section{Discussion}

True aneurysmal degeneration of the brachial artery is rare, with an incidence of $0.17 \%$. It may be associated with infection,

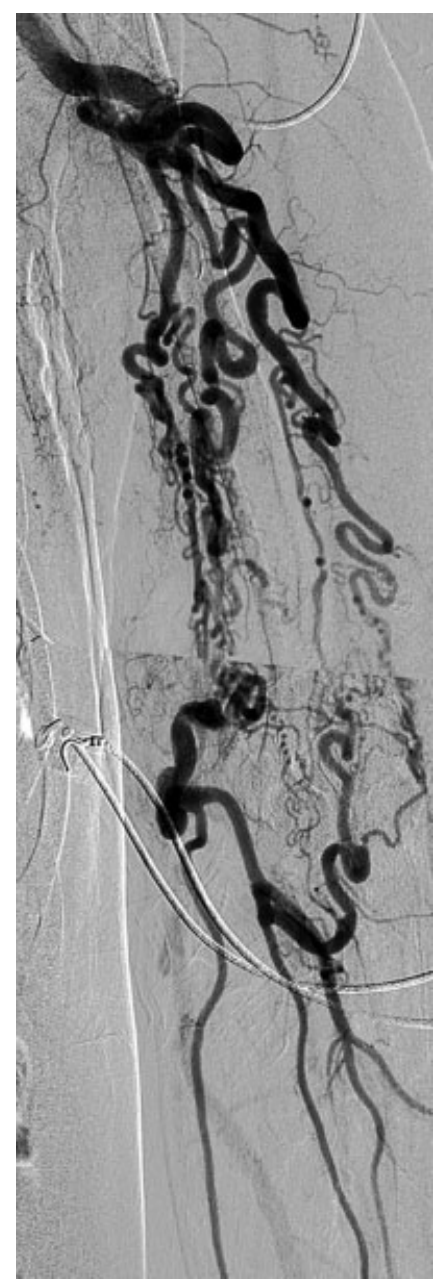

Fig. 2 Digital subtraction angiography showing occlusion of brachial artery secondary to an aneurysm thrombosis. 


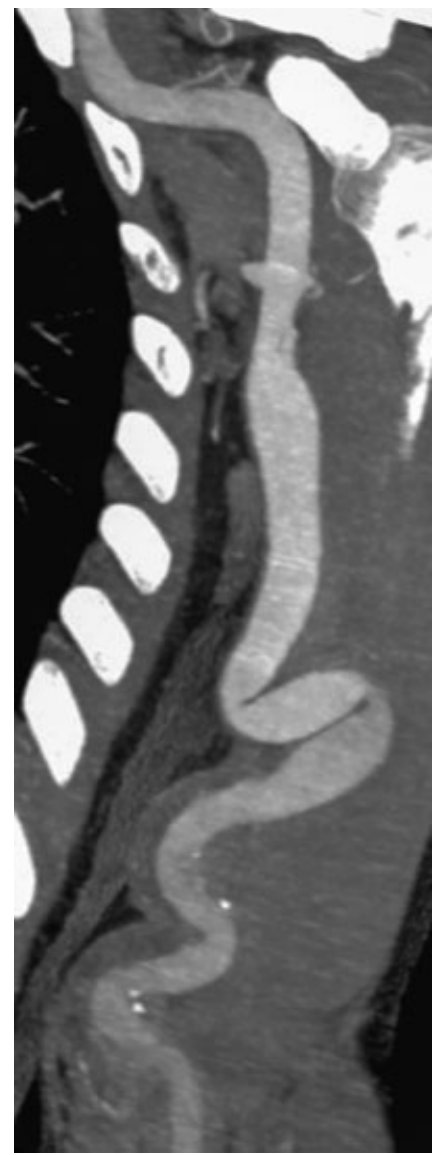

Fig. 3 Brachial artery aneurysm (angio-CT scan reconstruction). CT, computed tomography.

trauma, congenital defects, or iatrogenic injuries. ${ }^{6,9}$ Most reported cases of true brachial artery aneurysms have been a consequence of longstanding posttraumatic AVFs. ${ }^{10}$ Aneurysms can also develop in the inflow arteries after construction of AVFs for hemodialysis. ${ }^{4-6}$

Several studies have described an increase in the diameter of the inflow artery after construction of an AVF in their extremity. ${ }^{4-6,8,10}$ Arterial "growing" may be accentuated by AVF flow and the time after fistula creation. ${ }^{4,8}$ There are four pathophysiological steps that can explain the transition from arteriomegaly to aneurysmal degeneration. ${ }^{6}$ First, upregulation of local production of vasodilator agents (nitric oxide) and matrix metalloproteinases 2 and 9, which results in loss of vessel's wall integrity and aneurysmal remodeling. ${ }^{1-13}$ Second, degradation of elastic fibers and higher amounts of calcium and phosphate deposition (increased in dialysis patients), with reduction in the ability of vessel's wall to vasoconstrict. ${ }^{11-14}$ Third, the long-term use of immunosuppression and corticosteroid therapy in renal transplantation patients, that culminates in negative remodeling, due to the activation of a proinflammatory process. ${ }^{15-17}$ Finally, increased resistance of the vessel's wall, after ligation of the AVF, which weakens it and eventually leads to aneurysmal dilatation. $^{8}$

The prevalence of DBAA in patients with arteriovenous accesses is difficult to determine. The rarity of these aneur- ysms when compared with the number of AVFs created worldwide may be explained by the limited life expectancy of patients with end-stage renal disease undergoing hemodialysis, which until recent years was not long enough to permit the occurrence of aneurysmal degeneration. ${ }^{4}$

Donor artery aneurysms can develop from a few days to years after AVF creation. ${ }^{4,5}$ In our series, the onset time of brachial aneurysms varied from 64 to 288 months, with a mean time of 136.7 months. Previous reports showed similar results. $^{4-6}$

All of the brachial artery aneurysms from our series were diagnosed after closure or thrombosis of previous ipsilateral AVFs. Basile et al showed that vascular access closure does not stop the development of DBAA. ${ }^{18}$ Brachial artery aneurysms can appear years after AVF ligation. ${ }^{9,10}$

The most common clinical presentations of DBAA are pain, swelling, and pulsatile mass in the arm. Distal embolization can occur in almost $30 \%$ of cases, due to mobilization of mural thrombus within the aneurysm. ${ }^{4}$ Nerve compression/paresthesia has been described occasionally and rupture is extremely rare., ${ }^{4,5}$ In our series, all patients were symptomatic at the time of diagnosis. Our findings are in consonance with the literature. The majority of patients (60\%) presented with localized pain and pulsatile mass, and distal embolization occurred in $30 \%$ of the cases.

According to previous reports, duplex scan was the primary and almost the only investigative modality. ${ }^{6,19}$ CT scan angiography, due to limitations of contrast use in renal failure or renal transplant patients, was used only in selected cases. We used angiography to characterize forearm runoff, when distal embolization was present or when distal arteries were involved by diffuse atherosclerotic disease.

Surgical treatment by aneurysmectomy and interposition bypass is, in our opinion, a safe and effective option. The results from our series are good: our patients did not develop thrombosis of any interposition bypass, and during the entire follow-up period, only one patient developed an anastomotic stenosis.

We used autogenous conduits whenever possible. Reported series also prefer autogenous conducts. ${ }^{4-6,19}$ Mestres et al defend the use of ipsilateral arm vein, when not dilated or altered by previous AFV. ${ }^{19}$ This allows a quicker procedure with lower morbidity and preserves lower limb veins for future vascular procedures. In the present series, we also used ipsilateral arm veins, with good results.

We think that it is important to maintain follow-up of treated patients. There is a persistent risk of developing other donor artery aneurysms and risk of degeneration of the proximal and distal landing zones of the bypass. In addition, arteriomegaly may make it more difficult to find safe proximal and distal "nonaffected" artery for anastomotic sites ${ }^{20}$. Marzelle et al reported some cases of patients with synchronous donor artery aneurysms. ${ }^{4}$

This work presents one of the largest reported series of treated DBAA after AVF creation. Chemla et al reported treatment of 13 patients with brachial artery aneurysms ( 6 autogenous interposition bypass and 7 end-to-end anastomosis; mean follow-up of 14 months) and Martins et al reported 
treatment of 6 patients ( 4 autogenous interposition bypass, 1 prosthetic bypass, and 1 end-to-end anastomosis; mean follow-up of 35 months). ${ }^{5,21}$ Kordzadeh et al, in a systematic review of donor artery aneurysms following AVF ligation, reported 23 cases of brachial artery aneurysms. ${ }^{6}$ Our series presents the largest number of autogenous interposition bypass use for treatment of DBAA reported in the literature. Our series also presents the longest mean follow-up period (69 months).

Systematic duplex screening of donor artery aneurysms is not defended by some authors because in most cases, aneurysms can be diagnosed by physical examination. ${ }^{19}$ However, it is important to be aware of the risk of developing donor brachial aneurysms, particularly in certain groups of patients: patients with renal transplant or under immunosuppression and patients with longstanding and/or high flow AVFs.

\section{Conclusion}

The pathogenesis underlying donor artery aneurysmal degeneration remains unclear. Increased blood flow after AVF creation, immunosuppressive therapy, and ligation/thrombosis of the AVF may contribute to aneurysm formation.

Surgical treatment by aneurysmectomy and bypass, using autogenous grafts, is a safe and effective option.

\section{References}

1 Gilmore J. KDOQI clinical practice guidelines and clinical practice recommendations-2006 updates. Nephrol Nurs J 2006;33(5): 487-488

2 Vascular Access Work Group. Clinical practice guidelines for vascular access. Am J Kidney Dis 2006;48(Suppl 1):S248-S273

3 Hunter W. The history of an aneurysm of the aorta with some remarks on aneurysms in general. Trans Med Obstet Soc Phys Lond 1757; $1: 323$

4 Marzelle J, Gashi V, Nguyen HD, Mouton A, Becquemin JP, Bourquelot P. Aneurysmal degeneration of the donor artery after vascular access. J Vasc Surg 2012;55(4):1052-1057

5 Chemla E, Nortley M, Morsy M. Brachial artery aneurysms associated with arteriovenous access for hemodialysis. Semin Dial 2010;23(4):440-444

6 Kordzadeh A, D'Espiney Barbara RM, Ahmad AS, Hanif MA, Panayiotopoulos YP. Donor artery aneurysm formation following the ligation of haemodialysis arteriovenous fistula: a systematic review and case reports. J Vasc Access 2015;16(1):5-12
7 Johnston KW, Rutherford RB, Tilson MD, Shah DM, Hollier L, Stanley JC. Suggested standards for reporting on arterial aneurysms. Subcommittee on Reporting Standards for Arterial Aneurysms, Ad Hoc Committee on Reporting Standards, Society for Vascular Surgery and North American Chapter, International Society for Cardiovascular Surgery. J Vasc Surg 1991;13(3):452-458

8 Eugster T, Wigger P, Bölter S, Bock A, Hodel K, Stierli P. Brachial artery dilatation after arteriovenous fistulae in patients after renal transplantation: a 10-year follow-up with ultrasound scan. J Vasc Surg 2003;37(3):564-567

9 Dinoto E, Bracale UM, Vitale G, et al. Late, giant brachial artery aneurysm following hemodialysis fistula ligation in a renal transplant patient: case report and literature review. Gen Thorac Cardiovasc Surg 2012;60(11):768-770

10 Mellière D, Hassen-Khodja R, Cormier JM, Le Bas P, Mikati A, Ronsse H. Proximal arterial dilatation developing after surgical closure of long-standing posttraumatic arteriovenous fistula. Ann Vasc Surg 1997;11(4):391-396

11 Roy-Chaudhury P, Spergel LM, Besarab A, Asif A, Ravani P. Biology of arteriovenous fistula failure. J Nephrol 2007;20(2):150-163

12 Chung AW, Yang HH, Kim JM, et al. Upregulation of matrix metalloproteinase-2 in the arterial vasculature contributes to stiffening and vasomotor dysfunction in patients with chronic kidney disease. Circulation 2009;120(9):792-801

13 Chung AW, Yang HH, Sigrist MK, et al. Matrix metalloproteinase2 and -9 exacerbate arterial stiffening and angiogenesis in diabetes and chronic kidney disease. Cardiovasc Res 2009; 84(3):494-504

14 Martin BJ, Stehbens WE, Davis PF, Ryan PA. Scanning electron microscopic study of hemodynamically induced tears in the internal elastic lamina of rabbit arteries. Pathology 1989;21(3):207-212

15 Ihara M, Urata H, Kinoshita A, et al. Increased chymase-dependent angiotensin II formation in human atherosclerotic aorta. Hypertension 1999;33(6):1399-1405

16 Berger A. Th1 and Th2 responses: what are they? BMJ 2000; 321(7258): 424

17 Mitchell RN, Libby P. Vascular remodeling in transplant vasculopathy. Circ Res 2007;100(7):967-978

18 Basile C, Antonelli M, Libutti P, Teutonico A, Casucci F, Lomonte C. Is there a link between the late occurrence of a brachial artery aneurysm and the ligation of an arteriovenous fistula? Semin Dial 2011;24(3):341-342

19 Mestres G, Fontsere N, Yugueros X, Tarazona M, Ortiz I, Riambau V. Aneurysmal degeneration of the inflow artery after arteriovenous access for hemodialysis. Eur J Vasc Endovasc Surg 2014;48(5):592-596

20 Kordzadeh A, Panayiotopoulos YP. Re: 'aneurysmal degeneration of the inflow artery after arteriovenous access for hemodialysis'. Eur J Vasc Endovasc Surg 2015;49(3):354

21 Martins P, Ferreira T, Manuel V, et al. Brachial artery aneurysm on receivers of kidney transplant. Angiol Cir Vasc 2014;10(2): $58-63$ 